Tsemo Aristide

College Boreal

1 Yonge Street, Toronto

tsemo58@yahoo.ca

\title{
Decomposition of groups and top couples.
}

\begin{abstract}
.
Recently, we have endowed various categories of groups with topologies. The purpose of this paper is to introduce on these categories others topologies which are statistically more suitable to study well-known problems in groups theory. We use this framework to define a notion of prime ideal and to provide a decomposition of a large class of groups into a product of prime Remark that a similar question has been studied in [5] by Kurata with innocent methods. We remark that these topologies can be extended to other categories like the categories of commutative algebras, associative algebras and left symmetric.
\end{abstract}

\section{Definition 1.}

A top couple $(C, D)$ is defined by:

A subcategory $C$ of the category of groups, a subclass $D$ of $C$ which satisfies the following properties:

T1. Let $G, G^{\prime}$ be objects of $C$ such that $G^{\prime}$ is in $D$, if there exists an injective morphism $i: G \rightarrow G^{\prime}$, then $G$ is in $D$.

T2. Let $G$ be an object of $D, I$ and $J$ two normal subgroups of $G$ such that $I \cap J=1$, then $I=1$ or $J=1$.

T3. Let $G$ be an object of $C$, the normal subgroup $I$ of $G$ is an ideal of $G$ if and only if the quotient $G / I$ is an object of $C$; we suppose that the inverse image of an ideal by a morphism of $C$ is an ideal.

\section{Remark.}

Let $[I, J]$ be the subgroup generated by the commutators $[x, y]=x y x^{-1} y^{-1}$. $x \in I$ and $y \in J$.In [12], we have defined a notion of Top couple where we have replaced the axiom T2 by the axiom T'2 as follows: Let $G$ be an object of $D$, $I, J$ two normal subgroups of $G,[I, J]=1$ implies $I=1$ or $J=1$; remark that $[I, J] \subset I \cap J$. This enables to obtain more examples of Top couples which are eventually commutative and non trivial. We start by our first example:

Let $G$ be a group, we denote by $C_{G}$ the comma category over $G$, the objects of $C_{G}$ are morphisms $f_{H}: G \rightarrow H$. We denote such an object by $\left(f_{H}, H\right)$. A morphism between $\left(f_{H}, H\right)$ and $\left(f_{L}, L\right)$ is a morphism of groups $f: H \rightarrow L$ such that $f \circ f_{H}=f_{L}$. Let $\left(H, \phi_{H}\right)$ be an object of $C(G)$ and $x$ an element of $H$, we denote by $G(x)$ the subgroup of $H$ generated by $\left\{g x g^{-1}, g \in G\right\}$. A non trivial element $x$ of $H$ is a divisor of zero if and only if there exists a non trivial element $y$ of $H$ such that $G(x) \cap G(y)=\{1\}$ and $[G(x), G(y)]=1$. We denote by $D_{G}$ the subcategory of $C_{G}$ whose objects are the objects of $C_{G}$ without divisors of zero. 
Proposition 1. The couple $\left(C_{G}, D_{G}\right)$ is a Top couple.

Proof. Let us verify the property T1: Let $H$ and $H^{\prime}$ be elements of $C_{G}$, suppose that $H^{\prime}$ is an object of $D_{G}$ and there exists an injective morphism $i$ : $H \rightarrow H^{\prime}$. If $x, y$ are elements of $H$ such that $G(x) \cap G(y)=1$ and $[G(x), G(y)]=$ 1 , we also have $G(i(x)) \cap G(i(y))=1$ and $[G(i(x)), G(i(y))]=1$ since $i$ is injective. Since $H^{\prime}$ does not have divisors of zero, we deduce that $i(x)=1$ or $i(y)=1$. This implies that $x=1$ or $y=1$ since $i$ is injective.

The verification of $\mathrm{T} 2$ :

Let $H$ be an object $D_{G}, I$ and $J$ two normal subgroups of $H$ such that $I \cap J=1$. Suppose that $I$ and $J$ are not trivial. Let $x$ be a non trivial element of $I$ and $y$ be a non trivial element of $J$, we have $G(x) \subset I$ and $G(y) \subset G$, this implies that $G(x) \cap G(y) \subset I \cap J=1$ and $[G(x), G(y)] \subset[I, J] \subset I \cap J=1$. Since $H$ does not have divisors of zero, we deduce that $x=1$ or $y=1$. This is a contradiction.

Verification of T3:

Let $f: H \rightarrow H^{\prime}$ be a morphism of $C_{G}$, and $I$ an ideal of $H^{\prime} ; f^{-1}(I)$ is an ideal of $H$ since we can endow $H / f^{-1}(I)$ with the structure induced by the morphism $p \circ f_{H}$, where $p: H \rightarrow H / f^{-1}(I)$ is the canonical projection.

Definitions 2. Let $(C, D)$ be a Top couple, and $H$ an object of $C$, an ideal $P$ of $H$ is prime if and only if $H / P$ is an object of $D$.

For every normal subgroup $I$ of $H$, we denote by $V_{H}(I)$ the set of prime ideals which contain $I$.

Proposition 2. Let $(C, D)$ be a Top couple and $H$ an object of $C$. For every normal subgroups $I, J$ of $H$, we have $V_{H}(I \cap J)=V_{H}(I) \cup V_{H}(J)$.

Let $\left(I_{a}\right)_{a \in A}$ be a family of normal subgroups of $H$, and $I_{A}$ the normal subgroup generated by $\left(I_{a}\right)_{a \in A}$, we have $V_{H}\left(I_{A}\right)=\cap_{a \in A} V\left(I_{a}\right)$.

Proof. Firstly, we show that $V_{H}(I \cap J)=V_{H}(I) \cup V_{H}(J)$. Let $P$ be an element of $V_{H}(I \cap J)$ suppose that $P$ does not contain neither $I$ nor $J$. Let $x \in I, y \in J$ which are not elements of $P$. We denote by $u(x)$ the normal subgroup of $H$ generated by $x$. We have $u(x) \cap u(y) \subset I \cap J \subset P$. This implies that $x \in P$ or $y \in P$.

Let $P \in V_{H}\left(I_{A}\right)$. For every $a \in A, I_{a} \subset I_{A} \subset P$. This implies that $P \subset \cap_{a \in A} V_{H}\left(I_{a}\right)$. Let $P \in \cap_{a \in A} V_{H}\left(I_{a}\right)$, for every $a \in A, I_{a} \subset P$; this implies that $I_{A} \subset P$.

\section{Remark.}

The space $\operatorname{Spec}_{G}(H)$ of prime ideals is endowed with a topology whose closed subsets are the subsets $V_{H}(I)$ and the empty subset of $H$.

Let $x$ and $y$ be divisors of zero in the $G$-group $H$; the subgroup of $H$ generates by $G(x)$ and $G(y)$ is isomorphic to the direct product $G(x) \times G(y)$.

This leads to the following definitions:

Definitions 3. Let $H$ be an element of $C_{G}$, the adjoint representation $A d: G \rightarrow \operatorname{Aut}(H)$ is the morphism which associates to $g \in G$ the automorphism of $H$ defined by $\operatorname{Ad}(g)(h)=g h g^{-1}, h \in H$. 
Let $H$ be an object of $C_{G}$ a non trivial subgroup $H^{\prime}$ of $H$ stable by the adjoint representation is $G$-decomposable if and only if there exists two non trivial subgroups $H_{1}$ and $H_{2}$ stable by the adjoint representations and an isomorphism of groups $f: H \rightarrow H_{1} \times H_{2}$ which commutes with the adjoint representation.

An object $H$ of $C_{G}$ is locally $G$-indecomposable if every non trivial subgroup of $H$ is not $G$-decomposable.

If $G$ is the trivial group, we will omit the suffix $G$ in the previous definitions, for example, we will speak of decomposable groups and locally indecomposable groups.

Proposition 3. A G-group $H$ does not have divisors of zero if and only if $H$ is locally $G$-indecomposable.

Proof. Suppose that the $G$-group $H$ does not have divisors of zero, let $L$ be a subgroup stable by the adjoint action; suppose that $L$ is isomorphic to the product of the non trivial subgroups $L_{1}$ and $L_{2}$ stable by the adjoint representation. Let $x_{1} \in L_{1}$ and $x_{2} \in L_{2}$ be non trivial elements; $\left(x_{1}, 1\right)$ and $\left(1, x_{2}\right)$ are divisors of zero. This is a contradiction.

Conversely, suppose that the $G$-group $H$ is locally indecomposable; let $x$ and $y$ be divisors of zero; the subgroup of $H$ generates by $G(x)$ and $G(y)$ is a subgroup of $G$ which is the direct product of the subgroups $G(x)$ and $G(y)$ which are stable by the adjoint action. This is a contradiction.

\section{Remark.}

Let $G$ be a group, to study the geometry of objects of $C_{G}$, it is very important to know objects without divisors of zero. Firstly, we are going to study these objects for $G=1$. We are also going to classify finitely generated nilpotent groups who do not have divisors of zero. Remark that finite groups without divisors of zero have been classified by Marin when $G=1$; to present his result, let us recall that the quaternionic group $Q_{n}$ ( $n$ is an integer superior or equal to 3 ) is a finite group of order $2^{n}$ with the presentation:

$$
<x, y: x^{2^{n-1}}=1, y^{2}=x^{2^{n-2}}, y^{-1} x y=x^{-1}>
$$

Theorem Marin [6]. Suppose that $G=1$; a finite group $H$ is indecomposable if and only if:

1. $H$ is isomorphic to $Z / p^{n}$ for some prime $p$.

2. $H$ is isomorphic to $Q_{n}, n \geq 3$.

3. $H$ is isomorphic to an extension of $Z / q^{b}$ by $Z / p^{a}$ where $p$ and $q$ are different prime integers such that $p$ is odd, $q^{b}$ divides $p-1$ and the image of $Z / q^{b}$ in $\left(Z / p^{a}\right)^{*}$ has order $q^{b}$.

Proposition 4. Suppose that $G=1$, let $H$ be a group without divisors of zero. The rank of every commutative subgroup of $H$ is inferior to 1 . In particular the rank of the center $C(H)$ is inferior to 1 . If the center is not trivial, for every $y \in H$, there exists $n \in N$ such that $y^{n}$ is an element of $C(H)$ distinct of the identity. If the order of the center $C(H)$ is finite, then the order 
of every element of $H$ is finite and in this case the order of such an element is $p^{n}$ where $p$ is a prime integer.

Proof. If the rank of a commutative subgroup $L$ is strictly greater than 1 , there exists non trivial elements $x, y$ in $L$ such that $[x, y]=1$ and $(x) \cap(y)=1$. Where $(x)$ is the subgroup of $H$ generated by $x$. This is in contradiction with the fact that $H$ does not have zero divisors. Let $z \in C(H)$ be a non trivial element, for every element $x \in H$, we have $[x, z]=1$, since $H$ does not have divisors of zero, we deduce that $(x) \cap(z)$ is not the trivial group.

Suppose that the center of $H$ has a finite order, for any element $x \in H$, there exists an integer $n$ such that $x^{n} \in C(H), x^{n}$ and henceforth $x$ has a finite order. If the order of $z$ is the product $n m$ of two integers $n$ and $m$ which are relatively prime, then $z^{n}$ and $z^{m}$ are divisors of zero.

Theorem 1. Suppose that $G=1$, let $H$ be a finitely generated nilpotent group without divisors of zero. Then $H$ is finite or $H$ is isomorphic to $Z$.

Proof. Let $H$ be a non trivial finitely generated nilpotent group. recall that the derivative sequence of $H$ is defined by $H^{0}=H$, and $H^{(n)}=\left[H, H^{(n-1)}\right]$. There exists $n$ such that $H^{(n)}=1$, and $H^{(n-1)}$ is not trivial and contained in the center of $H$. The proposition 4 shows that the rank of $H^{(n-1)}$ is 1 . Suppose that there exists an element $x$ of $H^{(n-1)}$ which has a finite order, then every element of $H$ has a finite order. The subgroup $H^{(n-2)}$ is finite since it is the extension of a commutative finite group by a commutative finite group; recursively, we obtain that $H$ is finite.

Suppose now that $C(H)$ has infinite order and the rank of $H$ is different of 1. We have $\left[H, H^{(n-2)}\right]=H^{(n-1)}$. This implies the existence of an element $x \in H$ and $y \in H^{(n-2)}$ such that $[x, y] \in H^{(n-1)}$ and is distinct of the neutral element and has an infinite order. Remark that $[x, y]=h$ is in the center of $H$. There exists integers $n, m$ such that $x^{n} \in C(H)$ and $y^{m} \in C(H)$. We have $x^{n} y^{m} x^{-n} y^{-m}=h^{m n} y^{m} x^{n} x^{-n} y^{-m}=1$. This implies that the order of $h$ is finite. This is a contradiction with the hypothesis.

Corollary 1. A finitely generated locally indecomposable whose commutator subgroup is nilpotent is a finite group or is a finite extension of $Z$.

Proof. Let $H$ be a finitely generated locally indecomposable whose commutator subgroup is nilpotent. Then $[H, H]$ is a locally indecomposable nilpotent group. Suppose that $[H, H]$ is infinite, thus $[H, H]=Z$. Let $x$ be an element of $H ; A d_{x}:[H, H] \rightarrow[H, H]$ defined by $A d_{x}(y)=x y x^{-1}$ has order inferior to 2 since the group of automorphisms of $Z$ is isomorphic to $Z / 2$. Let $y$ be a non trivial element of $[H, H]$, we deduce that for every $x \in H,\left[x^{2}, y\right]=1$. Since $H$ does not have divisors of zero, it results that there exists $n, m$ such that $x^{2 n}=y^{m}$. Thus the quotient $H /[H, H]$ is finite.

Suppose that $[H, H]$ is finite and for every $x \in H, A d_{x}$ is an automorphism of a finite group, thus there exists $n$ such that $A d_{x^{n}}$ is the identity. Let $z$ be a non trivial element of $[H, H],\left[x^{n}, z\right]=1$, since $H$ does not have divisors of zero, we deduce that there exists $m$ such that $x^{n m} \in[H, H]$; thus every element of $H$ has a finite order. Since $H$ is solvable, we deduce that $H$ is finite. 
Corollary 2. A subgroup I of a finitely generated commutative group $H$ is a prime ideal if and only if $G / H$ is isomorphic either to $Z$ or to $Z / p^{n}$ where $p$ is a prime.

Proof. Let $I$ be a prime ideal of the finitely generated commutative group $H$, if $H / I$ is finite, Marin implies that $H / I$ is isomorphic to $Z / p^{n}$ where $n$ is a prime if $H / I$ is infinite, since it nilpotent, proposition implies that $H$ is isomorphic to $Z$.

Remark.

Suppose that $H=Z$ the group of relative integers. Let $I$ be a ideal of $H$, we know that $I$ is a subgroup generated by a positive integer $n$, write $n=\prod_{i \in I} p_{i}^{n_{i}}$. Let $p$ be a prime number and $a$ and integer, the prime ideal $\left(p^{a}\right)$ generated by $p^{a}$ is an element of $V((n))$ if and only if $p^{a}$ divides $n$.

We are going to present other examples of locally indecomposable groups Recall that the Tarski group is an infinite group $H$ such that there exists a prime integer $p$ such that every subgroup of $H$ is isomorphic to the cyclic group $Z / p$. The Tarski group is known to be simple. Olshans'kii [8] and have shown the existence of Tarski groups for $p>10^{75}$.

Adyan and Lysenok [1] and have generalized the construction of Ovshan'skii and shown that for $n>1003$ there exists non commutative groups $H$ such that every proper subgroup of $H$ is isomorphic to a subgroup isomorphic to $Z / n$, we will call these groups Adyan-Lysenok groups.

Remark that the Adyan-Lysenok groups $H$ defined for $n=p^{m}$ is a domain for $G=1$ : Let $x, y$ divisors of zero in $H$, since the subgroup $<x, y\rangle$ generated by $x$ and $y$ is a commutative subgroup we deduce that $\langle x, y\rangle$ is isomorphic to a subgroup of $Z / p^{m}$. This is in contradiction with the fact that $\langle x\rangle \cap\langle y\rangle$ is trivial.

More domains can be constructed by using the following proposition:

Proposition 5. The free product two locally indecomposable groups is a locally indecomposable group.

Proof. Let $G$ and $H$ be two locally indecomposable groups. Let $x$ and $y$ be divisors of zero, then since $x y=y x$, the corrollary [7] 4.1.6 p.187 shows either:

- $x$ and $y$ are conjugated in the same factor of $G$ or $H$. This is impossible since $G$ and $H$ are locally indecomposable

- $x$ and $y$ are the power of the same element. This is in contradiction with the fact that $x$ and $y$ are divisors of zero.

Definition 4. Let $H$ be an element of $C(G)$, we denote by $\operatorname{Rad}_{G}(H)$ the intersection of all the prime ideals of $H$.

Recall that a topological space $X$ is irreducible if and only if it is not the union of two proper subsets.

We say that an ideal $I$ is a radical ideal if it is the intersection of all the prime which contains $I$.

Proposition 6. Let $H$ be an element of $C(G)$, and $I$ a radical ideal of $H$, then $V_{H}(I)$ is irreducible if and only if $I$ is a prime. 
Proof. Suppose that $I$ is a prime, and $V_{H}(I)=V_{H}(J) \cup V_{H}(K)$ where $V_{H}(J)$ and $V_{H}(K)$ are proper subsets, since $I$ is a prime, $I$ is an element of $V_{H}(I)$. This implies that $I \in V_{H}(J)$ or $V_{H}(K)$. If $I$ is an element of $V_{H}(J)$, then $V_{H}(I) \subset V_{H}(J)$; if $I \in V_{H}(K)$, then $V_{H}(I) \subset V_{H}(K)$.This is a contradiction with the fact that $V_{H}(J)$ and $V_{H}(K)$ are proper subsets of $V_{H}(I)$.

Suppose that $V_{H}(I)$ is irreducible; let $x, y$ be elements of $H$ such that $[G(x), G(y)] \subset I$ and $G(x) \cap G(y) \subset I$. Let $u(x)$ be the normal subgroup generated by $x, u(x) \cap u(y)$ and $[u(x), u(y)]$ are contained in $I$. Since $V_{H}(u(x) \cap u(y))=$ $V_{H}(u(x)) \bigcup V_{H}(u(y))$, this implies that $V_{H}(I)=V_{H}(u(x)) \cap V_{H}(I) \bigcup V_{H}(u(y)) \cap$ $V_{H}(I)$. Since $V_{H}(I)$ is irreducible, we deduce that $V_{H}(I)$ is contained in $V_{H}(u(x))$ or is contained in $V_{H}(u(y))$. If $V_{H}(I)$ is contained in $V_{H}(u(x))$, the $\cap_{P \in V_{H}(I)} P=$ $I$ contains $u(x)$. It results that $x \in I$ since $I$ is a radical ideal. Similarly, if $V_{H}(I) \subset V_{H}(u(y))$ we deduce that $y \in I$.

Definition 5. Recall that a space is Noetherian if and only if every ascending chain of closed subsets $Z_{0} \subset Z_{1} \ldots \subset Z_{n} \subset \ldots$ stabilizes, this is equivalent to saying that there exists $i$ such that for every $n>i, Z_{n}=Z_{i}$. We deduce that the topological space $\operatorname{Spec}_{G}(H)$ is Noetherian if and only if a descending chain of normal subgroups of $H\left(I_{n}\right)_{n \in N}$ such that $I_{n+1} \subset I_{n}$ stabilizes.

\section{Remark.}

Let $G$ be a group:

$G$ is an element of $\operatorname{Spec}_{G}(G)$, we denote by $\operatorname{Spec}_{G}(G)^{*}, \operatorname{Spec}_{G}(G)-\{G\}$. We will denote by $V_{H}^{*}(I)$, the intersection $V_{H}(I) \cap \operatorname{Spec}_{G}(G)^{*}$.

For every element $x \in G, G(x)$ is the normal subgroup generated by $x$.

A maximal normal subgroup $I$ of $G$ is a prime, since $G / I$ is a simple group.

Theorem 2. Suppose that $\operatorname{Spec}_{G}(G)^{*}$ is Noetherien and $\operatorname{Rad}_{G}(G)=1$, then $G$ is the product of groups $G_{1} \times \ldots \times G_{n}$ such that for every $i$, the subgroup $H_{i}$ of $H$ generated by $G_{j}, j \in\{1, \ldots, n\}-\{i\}$ is a prime. Moreover, this decomposition is unique up to the permutation of the $G_{i}$.

Proof. Suppose that $\operatorname{Spec}_{G}(G)^{*}$ is Noetherian, then $\operatorname{Spec}_{G}(G)^{*}$ is the disjoint union of closed subsets $\left(V_{G}\left(H_{i}\right)\right)_{i=1, \ldots, n}$.

The intersection $\cap_{i=1, \ldots, n} H_{i}=1$. This is due to the fact that $V_{G}^{*}\left(\cap_{i=1, \ldots, n} H_{i}\right)=$ $V_{G}^{*}\left(H_{1}\right) \cup \ldots \cup V_{G}^{*}\left(H_{n}\right)=\operatorname{Spec}_{G}^{*}(G)$ and $\operatorname{Rad}_{G}(G)=1$.

We write $G_{i}=\cap_{j \in\{1, \ldots, n\}-\{i\}} H_{j}$. We are going to show that $G$ is isomorphic to the direct product $G_{1} \times \ldots \times G_{n}$.

Firstly, remark that $G_{i} \cap G_{j}=\cap_{k=1, . ., n} H_{k}=1$ if $i \neq j$. Since the subgroup $G_{i}$ are normal, for $i \neq j$, we have $\left[G_{i}, G_{j}\right] \subset G_{i} \cap G_{j}=1$. This implies that the subgroup $L$ of $H$ generated by $\left.\left(G_{i}\right)_{\{} i=1, \ldots, n\right\}$ is isomorphic to the direct product $G_{1} \times G_{2} \times \ldots \times G_{n}$. It remains to shows that $G$ is equal to its subgroup $L$.

We have $V_{G}^{*}\left(G_{i}\right)=\bigcup_{j \in\{1, . ., n\}, j \neq i} V_{G}^{*}\left(H_{i}\right)$. This implies that $V_{G}^{*}(L)=\cap_{i=1, \ldots, n} \bigcup_{j \in\{1, . ., n\}, j \neq i} V_{G}^{*}\left(H_{i}\right)$ is empty. We deduce that $L=H$, otherwise $L$ would have been contained in a maximal ideal which would have been an element of $V_{G}^{*}(L)$.

We show now that the subgroup $L_{i}$ of generated by $\left(G_{j}\right)_{j \neq i}$ is $H_{i}$. For every $j \neq i, G_{j} \subset H_{i}$. Suppose that there exists an element $x \in H_{i}$ which is not in 
$L_{i}$. Since $H=G_{1} \times \ldots \times G_{n}$, we can write $=\left(x_{1}, \ldots, x_{n}\right), x_{j} \in G_{j}$ and $x_{i} \neq 1$, we have $x_{j} \in H_{i}, j \neq i$. This implies that $x_{i} \in H_{i}$. This is a contradiction since $H_{i} \cap G_{i}=\{1\}$

We show now that the decomposition is unique. Suppose that there are two decompositions $H=G_{1} \times \ldots \times G_{n}$ and $H=U_{1} \times \ldots \times U_{m}$ such that the group $H_{i}$ generated by $1 \times \ldots G_{j} \times 1 . ., j \neq i$ is a prime ideal, the group $L_{i}$ generated by $1 \times . . U_{j} \times \ldots \times 1 j \neq i$ is also a prime ideal. Then $\bigcup_{i=1, . ., n} V_{G}^{*}\left(H_{i}\right)$ and $\bigcup_{i=1, \ldots, m} V_{G}^{*}\left(L_{i}\right)$ are decomposition of $\operatorname{Spec}_{G}^{*}(G)$ as union of irreducible components. Since this decomposition is unique, we deduce that $n=m$, and up to permutation that $V_{G}^{*}\left(H_{i}\right)=V_{G}^{*}\left(L_{i}\right)$, since $U_{i}$ and $H_{i}$ are prime, we deduce that $H_{i}=L_{i}$. This implies that $G_{i} \simeq G / H_{i}$ and $U_{i} \simeq G / L_{i}$ are isomorphic.

Corollary 3. Suppose that $G$ is a finite group and $\operatorname{Rad}_{G}(G)=1$, then $G$ is a product of indecomposable subgroups.

\section{Some generalizations.}

Let $A$ be a commutative ring, in classical algebraic geometry a prime ideal $P$ of $A$ is an ideal $P$ such for every elements $a, b \in A, a b \in P$ implies that $a \in P$ or $b \in P$. Inspired by the topologies defined above, we define the following notion:

Definitions. Let $A$ be a ring non necessarily commutative, $a, b$ elements of $A$. We denote by $I(a)$ the two-sided ideal generated by $a$. A two-sided ideal of the ring $A$ is a $p$-prime if for every elements $a, b \in A, I(a) \cap I(b) \in P$ implies that $a \in P$ or $b \in P$.

Let $I$ be a two-sided ideal of $A$, we denote by $V(I)$ the set of prime ideals of $A$ which contain $I$.

Proposition. Let $I, J$ be two-sided ideals of $A$, we have: $V(I \cap J)=$ $V(I) \bigcup V(J)$. Let $\left(I_{a}\right)_{a \in A}$ be family of ideals of $A$ which generates the ideal $I_{A}$, we have $V\left(I_{A}\right)=\cap_{a \in A} V\left(I_{a}\right)$.

Proof. Firstly, we show that $V(I \cap J)=V(I) \bigcup V(J)$. Since $I \cap J \subset I$ and $I \cap J \subset J$, we have $V(I) \subset V(I \cap J)$ and $V(J) \subset V(I \cap J)$. Let $P$ be an element of $V(I \cap J)$, suppose that $P$ does not contain $I$ and $J$. Let $a \in I, b \in J$ be elements which are not in $P, I(a) \cap I(b) \subset I \cap J$. This is a contradiction since $P$ is a prime ideal.

Let $P$ be an element of $V\left(I_{A}\right)$, since $I_{A} \subset P, I_{a} \subset P$ for every $a \in A$, this implies that $P \in \cap_{a \in A} V\left(I_{a}\right)$. Conversely, let $P \in \cap_{a \in A} V\left(I_{a}\right)$, for every $a \in A$, $I_{a} \subset P$. This implies that $I_{A} \subset P$.

\section{Examples.}

Suppose that $A$ is a commutative algebra, an ideal $I$ is a prime if and only if for every $a, b \in A, I(a) \cap I(b) \subset P$ implies that $a \in P$ or $b \in P$. This structure is different from the classical notion of prime. As we have seen, if $A=Z, Z / p^{n}$ is a prime ideal. 


\section{References.}

1. Adyan S.I. Lysenok I.G., On groups all of whose proper subgroups of which are finite cyclic, Izv. AN SSSR. Ser. matem., 55:5 (1991), 933990

2. Amaglobeli. M.G Algebraic sets and coordinate groups for a free nilpotent group of nilpotency class 2. Sibirsk. Mat. Zh. Volume 48 p. 5-10.

3. Baumslag, G, Miasnikov, A. Remeslennikov, V.N. Algebraic geometry over groups I. Algebraic sets and ideal theory. J. Algebra. 1999, 219, 1679.

4.. A.Grothendieck, Él'ements de géométrie algébrique I.Publications mathématiques de l'I.H.E.S 4, 5-228

5. Kurata, Y. A decomposition of normal subgroup in a group. Osaka J. Math. 1 (1964), 201-229

6. Marin, I. Strongly indecomposable finite groups Expositiones Mathematicae, vol. 26, no. 3, pp. 261-267, 2008

7. Magnus, Karass, Solitar. Combinatorial group theory, Dover publication 1976

8. Olshanskii A., Groups of bounded period with subgroups of prime order, Algebra and Logic 21 (1983), 369-418; translation of Algebra i Logika 21 (1982), 553-618

9. Scott W.R Algebraically closed groups Proc. Amer. Math. Soc. 2 (1951) $118-121$

10. Serre J-P. (GAGA) Géométrie algébrique, géométrie analytique, Annales de l'Institut Fourier, Grenoble t. 6 1955-1956 1-42.

11. Tsemo, A. Scheme theory for groups and Lie algebra, International Journal of Algebra 5. 2011 139-148

12. Tsemo, A. Some properties of schemes in groups theory and Top couples International Journal of Algebra, Vol. 7, 2013, no. 1, 25 - 48.

13. Tsemo, A. Theory of curves, in preparation. 